University of New Orleans

ScholarWorks@UNO

$11-15-1987$

\title{
Constraint on the optical constants of a transparent film on an absorbing substrate for inversion of the ratio of complex $p$ and $s$ reflection coefficients at a given angle of incidence
}

R. M.A. Azzam

University of New Orleans, razzam@uno.edu

M. A. Habli

Follow this and additional works at: https://scholarworks.uno.edu/ee_facpubs

Part of the Electrical and Electronics Commons

\section{Recommended Citation}

R. M. A. Azzam and M. A. Habli, "Constraint on the optical constants of a transparent film on an absorbing substrate for inversion of the ratio of complex $\mathrm{p}$ and $\mathrm{s}$ reflection coefficients at a given angle of incidence," Appl. Opt. 26, 4717-4721 (1987)

This Article is brought to you for free and open access by the Department of Electrical Engineering at ScholarWorks@UNO. It has been accepted for inclusion in Electrical Engineering Faculty Publications by an authorized administrator of ScholarWorks@UNO. For more information, please contact scholarworks@uno.edu. 


\title{
Constraint on the optical constants of a transparent film on an absorbing substrate for inversion of the ratio of complex $p$ and $s$ reflection coefficients at a given angle of incidence
}

\author{
R. M. A. Azzam and M. A. Habli
}

\begin{abstract}
An absorbing substrate of complex refractive index $n_{2}-j k_{2}$ can be coated by a transparent thin film of refractive index $n_{1}$ and normalized thickness $\zeta$ so that the ratio of complex reflection coefficients for the $p$ and $s$ polarizations of the film-covered substrate $\rho$ is the inverse of that of the film-free substrate $\bar{\rho}$ at a given angle of incidence $\phi$. A pair of parallel (metallic) mirrors, one uncoated and the other coated with a $\rho$-inverting layer, causes a beam displacement without change of polarization and with a certain net reflectance (insertion loss) $\mathcal{R}$. In this paper the constraint on $n_{1}, n_{2}, k_{2}$ for $\rho$ inversion $(\rho \bar{\rho}=1)$ is represented by a family of constant $-n_{1}$ contours in the $n_{2} k_{2}$ plane at $\phi=45,60$, and $75^{\circ}$. Along each solution curve, $\zeta$ and $\mathcal{R}$ are also plotted vs $n_{2}$ at constant $n_{1}$. Analysis of the effect of small errors of incidence angles, film refractive index, and thickness is presented for two specific designs using $\mathrm{Al}$ mirrors at 650 and $950 \mathrm{~nm}$.
\end{abstract}

\section{Introduction}

At a given wavelength $\lambda$ and angle of incidence $\phi$, the change of the state of polarization of light on reflection from an uncoated absorbing (e.g., metallic) substrate is determined by the ratio

$$
\bar{\rho}=\bar{R}_{p} / \bar{R}_{s}
$$

of complex reflection coefficients $\bar{R}_{p}$ and $\bar{R}_{s}$ for the linear polarizations parallel $p$ and perpendicular $s$ to the plane of incidence. If the substrate is coated by a transparent thin film, the ratio is changed to

$$
\rho=R_{p} / R_{s} .
$$

By a suitable choice of film refractive index $N_{1}$ and thickness $d$, it is possible to make

$$
\rho=1 / \bar{\rho},
$$

i.e., $\rho$ can be inverted as has been shown recently. ${ }^{1}$ In functional form, the condition of $\rho$ inversion can be expressed as

\footnotetext{
The authors are with University of New Orleans, Department of Electrical Engineering, Lakefront, New Orleans, Louisiana 70148.

Received 16 March 1987.

0003-6935/87/224717-05\$02.00/0.

(C) 1987 Optical Society of America.
}

$$
\bar{\rho}\left(\phi, N_{2}\right) \rho\left(\phi, N_{1}, \zeta, N_{2}\right)=1,
$$

where $N_{2}=n_{2}-j k_{2}$ is the substrate complex refractive index and $\zeta$ is the film thickness as a fraction of the thickness period $D_{\phi}$ :

$$
\begin{aligned}
\zeta & =d / D_{\phi} ; \\
D_{\phi} & =\left(\lambda / 2 N_{0}\right)\left(N_{1}^{2}-\sin ^{2} \phi\right)^{-1 / 2} .
\end{aligned}
$$

$N_{1}$ and $N_{2}$ are normalized with respect to the refractive index $N_{0}$ of the transparent medium of incidence (usually air, $N_{0}=1$ ).

If a $\rho$-inverting layer is applied to one of the two parallel (metallic) mirrors of a beam displacer (Fig. 1), the state of polarization of light is preserved after two reflections. The insertion loss of such a device is given by the net two-bounce intensity reflectance $R$, which is the same for the $p$ and $s$ polarizations. Deviation from the exact condition of $\rho$ inversion is determined by the deviation of the ratio of net complex $p$ and $s$ reflection coefficients

$$
\rho_{n}=\bar{\rho} \rho
$$

from 1. This can be broken down to separate magnitude and phase errors:

$$
\text { mag. error }=\left|\rho_{n}\right|-1 \text {; phase error }=\arg \rho_{n} .
$$

In Ref. 1 Eq. (4) was considered as a constraint on $\phi, \zeta, N_{1}$ for two specific values of $N_{2}$, namely, 1.212$j 6.924(\mathrm{Al}$ at $0.6328 \mu \mathrm{m})$ and $9.5-j 73(\mathrm{Ag}$ at $10.6 \mu \mathrm{m})$. 


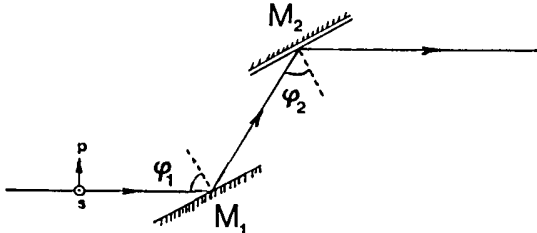

Fig. 1. Beam displacer of two parallel mirrors $M_{1}$ and $M_{2} . M_{2}$ is coated by a single transparent film to preserve polarization, and $M_{1}$ is left uncoated. $\phi_{1}$ and $\phi_{2}$ are the nominally equal angles of incidence at $M_{1}$ and $M_{2}$, respectively.

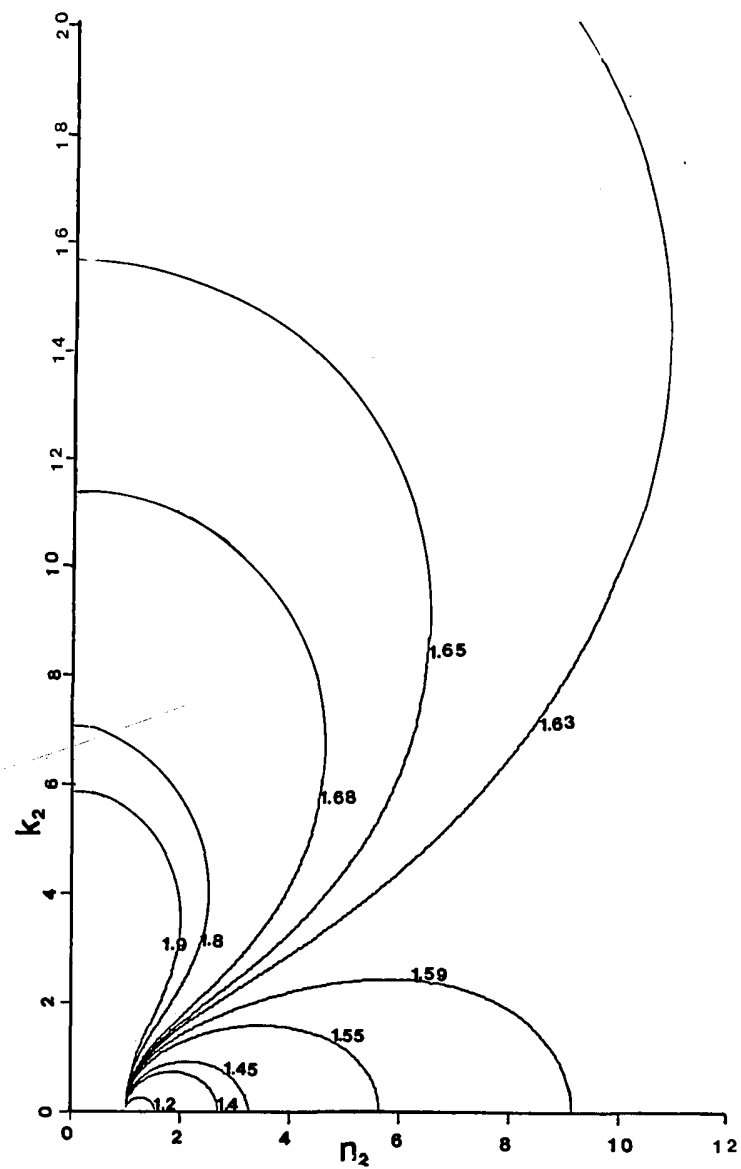

Fig. 2. Contours of constant film refractive index, $n_{1}=$ constant marked by each curve, in the $n_{2}, k_{2}$ plane, where $n_{2}-j k_{2}$ is the substrate complex refractive index. These equi- $n_{1}$ contours represent the constraint on the optical constants so that the ratio of the complex $p$ and $s$ reflection coefficients of the film-substrate system $\rho$ is the inverse of the corresponding ratio $\bar{\rho}$ of the uncoated substrate (i.e., $\rho=1 / \bar{\rho}$ ) at the same angle of incidence $\phi=45^{\circ}$.

In this paper we consider $\rho$ inversion at a given angle of incidence over a wide range of substrates, corresponding to a rectangle of size $10 \times 20$ in the $n_{2} k_{2}$ plane, with the constraint on optical constants being represented by contours of constant $N_{1}$ in that plane. The variation of the associated normalized film thickness $\zeta$ and net reflectance $\mathcal{R}$ of the polarization-preserving paral- lel-mirror beam displacer (PPPMBD) with one coated mirror is determined along each $N_{1}=$ constant contour.

Finally, we conclude by an error analysis for two monochromatic designs using $\mathrm{Al}$ mirrors to show the effects of small errors of incidence angles, film refractive index, and thickness on the performance of such a system.

It should be noted that PPPMBD in which both mirrors are coated was considered earlier by Azzam ${ }^{2}$ and Azzam and Khan. ${ }^{3}$

\section{Constraint on Optical Constants for $\rho$ Inversion}

Equation (4) is complex and equivalent to two real constraints. For a given $\phi, \zeta$ can be separated from Eq. (4) leading to a constraint on the optical constants $N_{1}$ $=n_{1}$ and $N_{2}=n_{2}-j k_{2}$ of the form

$$
f\left(n_{1}, n_{2}, k_{2}\right)=0 .
$$

This separation-of-variables method is explained in Ref. 1 and is not repeated here. Equation (9) is represented by constant- $n_{1}$ contours in the $n_{2} k_{2}$ plane. To generate one such contour, $n_{1}$ is assigned a fixed value, $n_{2}$ is scanned over a certain range, and for each $n_{2}$ Eq. (9) is solved for $k_{2}$ as its only unknown by numerical iteration. Only $n_{1}$ values of $>1$ are taken (i.e., the film is assumed to be more optically dense than the medium of incidence), and we restrict ourselves to a rectangle of $10 \times 20$ size in the $n_{2} k_{2}$ plane (i.e., $0<n_{2} \lesssim 10,0<k_{2}<$ 20 ). The $n_{1}$ values are selected to generate reasonably well-spaced constant- $n_{1}$ contours in the $n_{2} k_{2}$ plane.

Figure 2 shows a family of equi- $n_{1}$ contours in the $n_{2} k_{2}$ plane for $n_{1}$ values (marked by each curve) from 1.2 to 1.9 representing the constraint on the film $\left(n_{1}\right)$ and substrate $\left(n_{2}, k_{2}\right)$ optical constants for $\rho$ inversion at $\phi=45^{\circ}$. All contours start from a common point $\left(n_{2}, k_{2}\right)=(1,0)$. Those for $n_{1} \leq 1.59$ terminate on the $n_{2}$ axis, and those for $n_{1} \geq 1.63$ terminate on the $k_{2}$ axis. Figure 2 suggests that a film with refractive index in the narrow interval $1.59<n_{1}<1.63$ inverts $\bar{\rho}$ of substrates represented by points in a large part of the first quadrant of the infinite $n_{2} k_{2}$ plane.

Figure 3 shows the variation of the normalized film thickness $\zeta$ required for $\rho$ inversion as a function of $n_{2}$ along each constant- $n_{1}$ contour of Fig. 2 . Notice that $\zeta$ $\rightarrow 0$ as $n_{2} \rightarrow 1$ and that $\zeta$ increases monotonically (notwithstanding the down-reading scale) toward $\zeta=$ $1 / 2$ as $n_{2}$ increases above 1 . $\zeta=1 / 2$ corresponds to a quarterwave optical thickness at oblique incidence; this is the required thickness of the $\rho$-inverting layer when the substrate is transparent $\left(k_{2}=0\right)$.

Figure 4 shows the two-bounce reflectance $R$ of the PPPMBD plotted vs $n_{2}$ with $n_{1}$ as a parameter along each constant- $n_{1}$ contour of Fig. 2 . It is evident that beam displacement with polarization preservation on double reflection, realized using a single-layer coating on one mirror, is accompanied by some insertion loss. For the range of $n_{2} k_{2}$ in Fig. 2, an insertion loss of $<3$ $\mathrm{dB}$ (i.e., $R>0.5$ ) requires a film with refractive index $n_{1}>1.6$ and a substrate with extinction coefficient $k_{2}$ $\gtrsim 3$. 


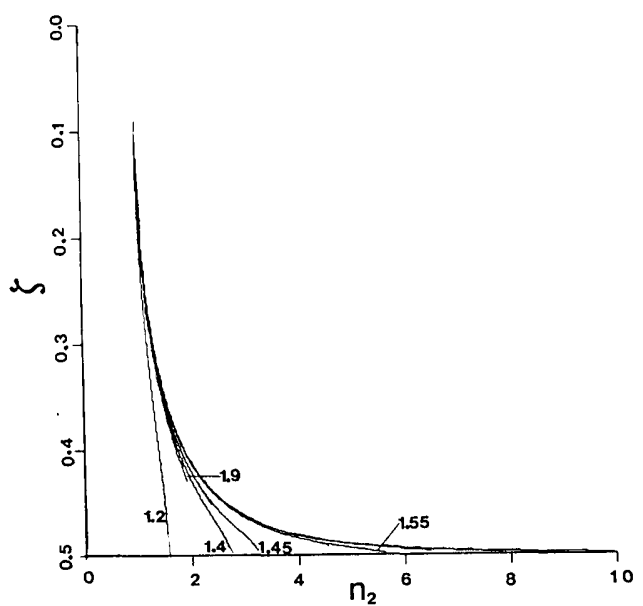

Fig. 3. Film thickness as a fraction of the film thickness period $\zeta$ required for $\rho$ inversion $(\rho=1 / \bar{\rho})$ at $\phi=45^{\circ}$ plotted as a function of $n_{2}$ at constant $n_{1}$ (marked by each curve) along each contour of Fig. 2.

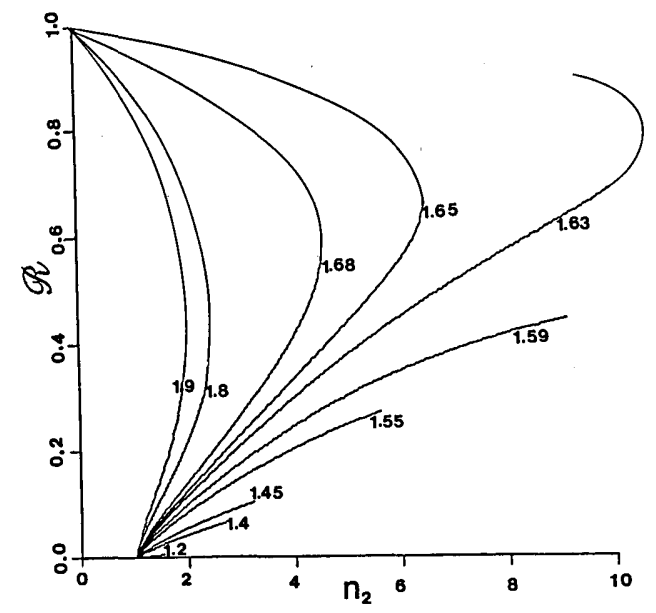

Fig. 4. Polarization-independent net reflectance $\mathscr{R}$ of the twomirror beam displacer of Fig. 1 when the condition $\rho \bar{\rho}=1$ is satisfied at $\phi_{1}=\phi_{2}=45^{\circ}$. $R$ is plotted as a function of $n_{2}$ at constant $n_{1}$ (marked by each curve) along each contour of Fig. 2 .

Figures 5-10 show additional results for angles of incidence of 60 and $75^{\circ}$, respectively. Except for a small compression of the range of $n_{1}$ at higher angles, the families of equi- $n_{1}$ contours in the $n_{2} k_{2}$ plane for $\phi$ $=45,60$, and $75^{\circ}$ are quite similar. The same applies to the contours of $\zeta$ vs $n_{2}$ at constant $n_{1}$ of Figs. 3, 6, and 9. Only the family of contours of $\mathcal{R}$ vs $n_{2}$ with an $n_{1}$ constant at $75^{\circ}$, Fig. 10 , is significantly different from the corresponding families at 45 and $60^{\circ}$ of Figs. 4 and 7.

\section{Error Analysis}

As specific examples, Table I lists two designs of PPPMBD using Al mirrors at wavelengths of 650 and $950 \mathrm{~nm}$ in the visible and near IR with incidence angles of 45 and $60^{\circ}$, respectively. The optical constants $n_{2}, k_{2}$ of $\mathrm{Al}$ are obtained from Ordal et al. ${ }^{4}$ The refrac-

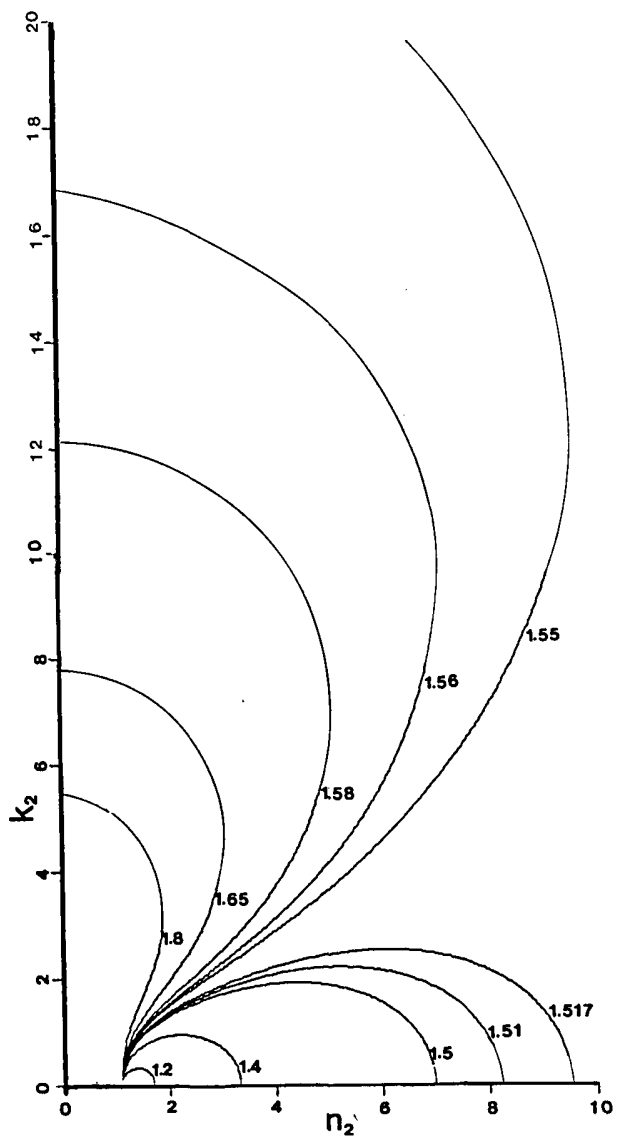

Fig. 5. Same as in Fig. 2 except that now $\phi=60^{\circ}$.

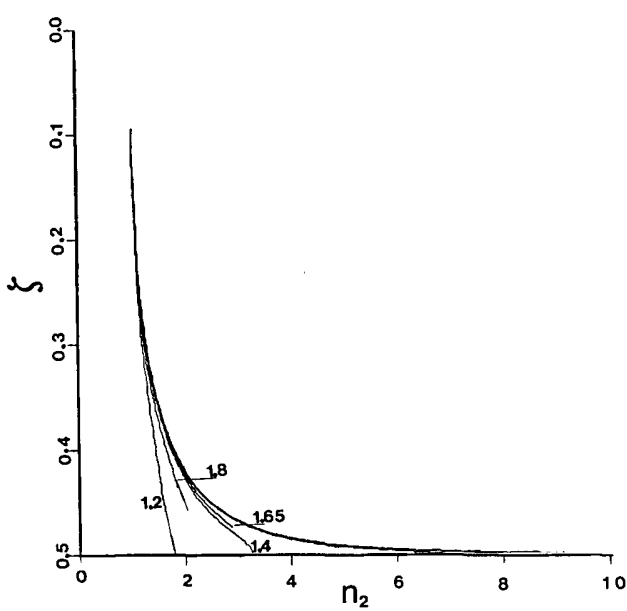

Fig. 6. Same as in Fig. 3 except that now $\phi=60^{\circ}$.

tive index $n_{1}$ of the transparent $\rho$-inerting layer on mirror 2 is calculated as described in Ref. 1 . The required indices of $\sim 1.8$ and 1.6 can be realized by coating materials such as $\mathrm{Sc}_{2} \mathrm{O}_{3}$ and $\mathrm{LaF}_{3}$ as given by Arndt et al. ${ }^{5}$ and Pulker, ${ }^{6}$ respectively. The twobounce net reflectance $\mathcal{R}$ of $\sim 66$ and $71 \%$ corresponds to an insertion loss of $\sim 2$ and $1.5 \mathrm{~dB}$, respectively, which is acceptable. ${ }^{7}$ 


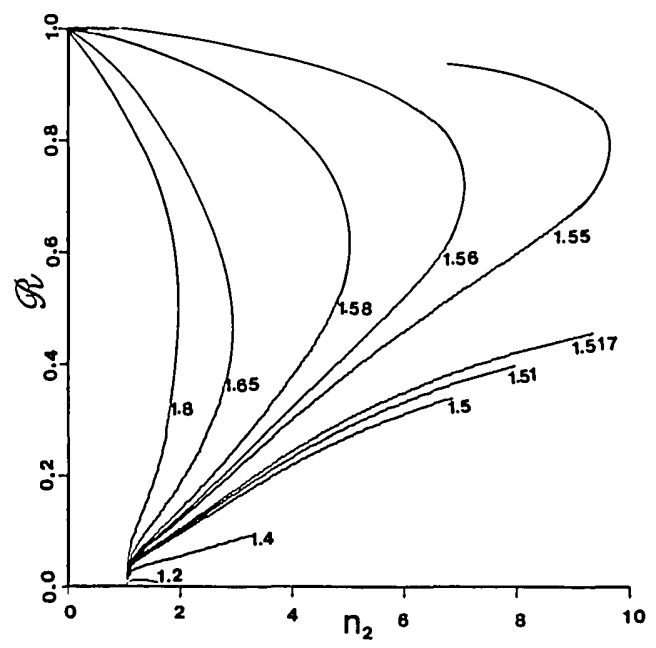

Fig. 7. Same as in Fig. 4 except that now $\phi_{1}=\phi_{2}=60^{\circ}$.

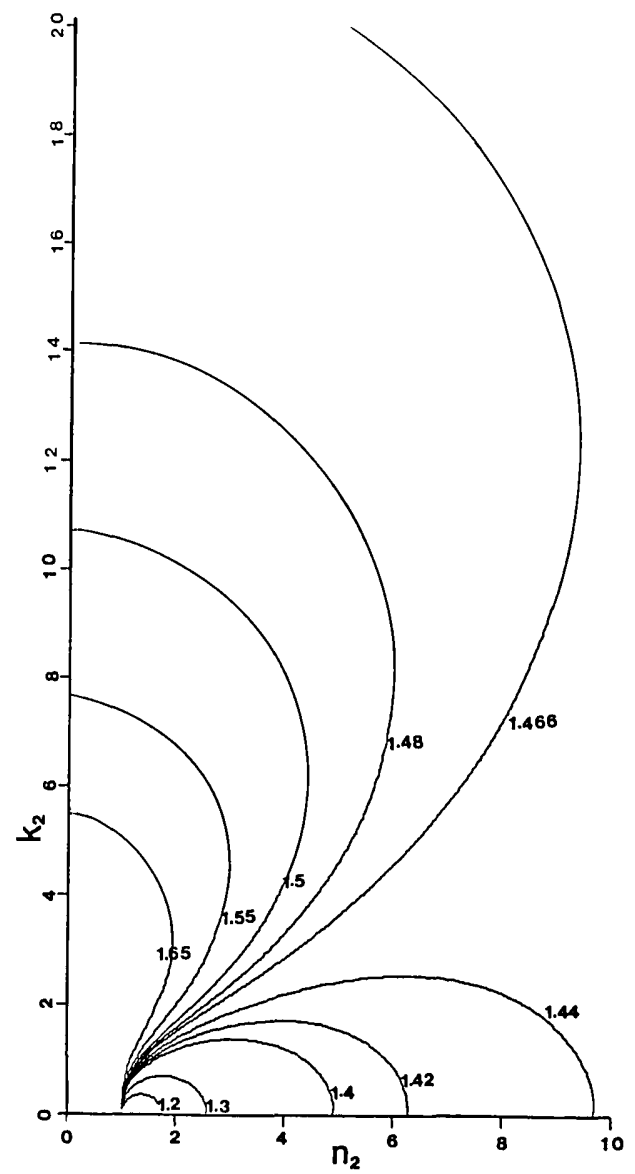

Fig. 8. Same as in Fig. 2 except that now $\phi=75^{\circ}$.

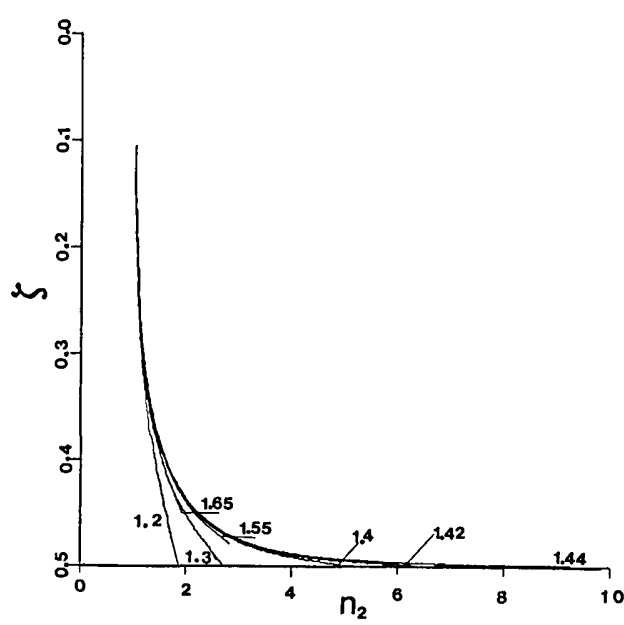

Fig. 9. Same as in Fig. 3 except that now $\phi=75^{\circ}$.

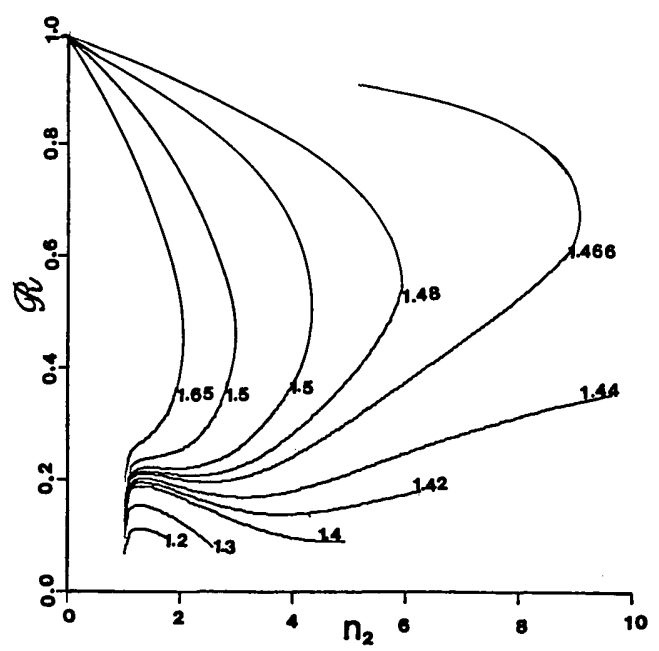

Fig. 10. Same as in Fig. 4 except that now $\phi_{1}=\phi_{2}=75^{\circ}$

Table I. Refractive Index $n_{1}$ and Thickness $d$ of a Transparent Film Coating on an Al Substrate of Complex Refractive-Index $n_{2}-j k_{2}$ Required to Invert $\rho$ at an Angle of Incidence $\phi^{a}$

\begin{tabular}{ccccccc}
\hline$\lambda(\mathrm{nm})$ & $n_{2}$ & $k_{2}$ & $n_{1}$ & $d(\mathrm{~nm})$ & $\phi(\mathrm{deg})$ & $\mathscr{R}(\%)$ \\
\hline 650 & 1.240 & 6.600 & 1.792047 & 95.59 & 45 & 65.9044 \\
950 & 1.750 & 8.500 & 1.616028 & 172.07 & 60 & 70.8287 \\
\hline
\end{tabular}

${ }^{a} \lambda$ is the wavelength of light, and $\mathcal{R}$ is the net polarizationindependent reflectance after two reflections at the same range $\phi$ from a pair of parallel $\mathrm{Al}$ mirrors, one of which is uncoated, and the second is coated with the $\rho$-inverting layer. Such a system functions as a polarization-preserving beam displacer.

Table II. Magnitude and Phase Errors that Result when Errors of Incidence Angles $\Delta \phi_{1}, \Delta \phi_{2}$, Film Refractive-Index $\Delta n_{1}$, and Film Thickness $\Delta d$ are Introduced one at a time in the Parallel-Mirror Beam Displacers whose Characteristics are Specifled in Table ${ }^{a}$

\begin{tabular}{|c|c|c|c|c|c|c|c|c|}
\hline \multirow[b]{2}{*}{$\lambda(\mathrm{nm})$} & \multicolumn{2}{|c|}{$\Delta n_{1}=0.01$} & \multicolumn{2}{|c|}{$\Delta d=1(\mathrm{~nm})$} & \multicolumn{2}{|c|}{$\Delta \phi_{1}=0.1(\mathrm{deg})$} & \multicolumn{2}{|c|}{$\Delta \phi_{2}=0.1(\mathrm{deg})$} \\
\hline & $\begin{array}{l}\text { Mag. } \\
\text { Error }\end{array}$ & $\begin{array}{c}\text { Phase } \\
\text { Error (deg) }\end{array}$ & $\begin{array}{l}\text { Mag. } \\
\text { Error }\end{array}$ & $\begin{array}{c}\text { Phase } \\
\text { Error (deg) }\end{array}$ & $\begin{array}{l}\text { Mag. } \\
\text { Error }\end{array}$ & $\begin{array}{c}\text { Phase } \\
\text { Error (deg) }\end{array}$ & $\begin{array}{l}\text { Mag. } \\
\text { Error }\end{array}$ & $\begin{array}{c}\text { Phase } \\
\text { Error (deg) }\end{array}$ \\
\hline 650 & $2.52 E-3$ & 0.5859 & $3.06 E-3$ & 1.2561 & $1.92 E-4$ & 0.0611 & $1.40 E-4$ & 0.1204 \\
\hline 950 & $4.60 E-3$ & 1.8772 & $2.15 E-3$ & 1.5260 & $3.17 E-4$ & 0.0945 & $2.68 E-4$ & 0.2380 \\
\hline
\end{tabular}

a The absolute values of the errors are indicated. $E-3$ is an abbreviated notation for $\times 10^{-3}$. 
Table II lists the magnitude and phase errors, Eqs. (8), caused by introducing one at a time angle-of-incidence errors $\Delta \phi_{1}, \Delta \phi_{2}$ of $0.1^{\circ}$, film-refractive-index error $\Delta n_{1}=0.01$, and film-thickness error $\Delta d=1 \mathrm{~nm}$. The first mirror is assumed to be the uncoated one (see Fig. 1) where the angle of incidence is $\phi_{1}$. These results indicate that the designs are reasonably tolerant to small angle errors (phase error $<0.25^{\circ}$, mag. error $<3.2 \times 10^{-4}$ ) but that the film refractive index and thickness should be tightly controlled to within 0.01 and $1 \mathrm{~nm}$, respectively, to avoid appreciable polarization errors.

We wish to thank A. El-Saba for his assistance. M. A. Habli is presently at the Department of Electrical Engineering and the Center for Applied Optics, University of Alabama in Huntsville.

\section{References}

1. R. M. A. Azzam, "Inverting the Ratio of the Complex Parallel and Perpendicular Reflection Coefficients of an Absorbing Substrate using a Transparent Thin-Film Coating," J. Opt. Soc. Am. A 1, 699 (1984).

2. R. M. A. Azzam, "Displacement of a Monochromatic Light Beam Parallel to Itself without Change of Polarization," Opt. Lett. 7, 80 (1982).

3. R. M. A. Azzam and M. E. R. Khan, "Polarization-Preserving Single-Layer-Coated Beam Displacers and Axions," Appl. Opt. 21, 3314 (1982).

4. M. A. Ordal et al., "Optical Properties of the Metals $\mathrm{Al}, \mathrm{Co}, \mathrm{Cu}$, $\mathrm{Au}, \mathrm{Fe}, \mathrm{Pb}, \mathrm{Ni}, \mathrm{Pb}, \mathrm{Pt}, \mathrm{Ag}, \mathrm{Ti}$, and $\mathrm{W}$ in the Infrared and Far Infrared," Appl. Opt. 22, 1099 (1983).

5. D. P. Arndt et al., "Multiple Determination of the Optical Constants of Thin Film Coating Materials," Appl. Opt. 23, 3571 (1984).

6. H. K. Pulker, "Characterization of Optical Thin Films," Appl. Opt. 18, 1969 (1979)

7. It is clear from Figs. 2, 5, and 8 and 4, 7, and 10, respectively, that higher net reflectances of PPPMBD are achieved with substrates, such as $\mathrm{Ag}$, with $n_{2}<1$ and $k_{2} \gg 1$
Patter continued from page 4708

ments at aspect ratios $<1.5$ and drop sizes less than $\sim 0.06$ of the sound wavelength.

This work was done by Eugene H. Trinh and Chaur-Jian Hsu of Caltech for NASA's Jet Propulsion Laboratory. Refer to NPO16746.

\section{Containerless atomic-fluorescence property measurements}

A report describes studies conducted to establish and verify the use of laser-induced fluorescence in monitoring and controlling high-temperature containerless processes. Specimens were levitated by gas jets or electromagnetic fields and heated by laser beams or electromagnetic induction while being irradiated and detected by the fluorescence technique. The reported work includes the development of an apparatus and its use in studies of the following phenomena:

Chemical reactions on $\mathrm{Al}_{2} \mathrm{O}_{3}, \mathrm{Mo}, \mathrm{W}$, and $\mathrm{LaB}_{6}$ specimens;

Methods for noncontact measurements of specimen temperature; Properties of levitating gas jets; and

Radiative lifetimes and collisional energy-transfer rates for electronically excited atoms.

A pulsed dye laser was used to induce fluorescence in atomic $\mathrm{Hg}$, $\mathrm{Al}, \mathrm{Mo}, \mathrm{W}, \mathrm{La}$, and $\mathrm{B}$. The $\mathrm{Hg}$ atoms were added to a jet of Ar. The $\mathrm{Al}$ atoms were produced by the continuous-wave laser heating of aerodynamically levitated sapphire and polycrystalline alumina spheres or of self-supported sapphire filaments. Mo atoms were evaporated from solid spheres that were inductively levitated in a vacuum and heated by a laser. $\mathrm{W}$ atoms were evaporated from electrically heated filaments. La and $B$ atoms were evaporated from an aerodynamically levitated and laser-heated $\mathrm{LaB}_{6}$ sphere.

The report makes quantitative and qualitative comparisons among three new methods of temperature measurement, all of which rely on laser-induced fluorescence. One method is gas-density thermometry with a seed gas (usually $\mathrm{Hg}$ ). This method requires an inert ambient atmosphere. The other two methods involve measurements of the velocities of evaporating atoms or of the population ratios of different electronic states. These methods require the assumption that the gas is in thermal equilibrium with the hot surface of interest and provide a check on the assumption in that when the assumption is not correct, they yield equal temperatures only by coincidence. The enthalpies of evaporation of $\mathrm{W}$ and Mo measured in these experiments agree, within experimental uncertainty, with the values in the literature. The evaluation of the $\mathrm{LaB}_{6-}$ evaporation results is complicated by theoretical difficulties in calculating radiative transitions of $\mathrm{B}$ atoms and the relationship between concentration and self-absorption of radiation. A further complication arises from the disagreement between earlier reported results and the results of these experiments. The discrepancies may be due in part to reactions with effusion cells.

The authors conclude that if accurate temperature measurements are taken, containerless measurements of laser-induced fluorescence, coupled with calculations based on the third law of thermodynamics, can lead to the accurate determinations of reaction enthalpies. For binary compounds, the required data are the activities of the elemental components, which can be determined directly from the ratios of the intensities of laser-induced fluorescence over the material of interest to those over the pure elements at the same temperature. Gas-phase/condensed-phase equilibrium is required for such determinations, and approximate equilibrium would be achieved over high-temperature liquids. Equilibrium can be further assured by the use of an inert-gas atmosphere to retard evaporation.

This work was done by P. C. Nordine, R. A. Schiffman, and C. A. Walker of Midwest Research Institute for Marshall Space Flight Center. Further information may be found in NASA CR171038[N84-25481/NSP], "Containerless High Temperature Property Measurements by Atomic Fluorescence." Copies may be purchased [prepayment required] from the National Technical Information Service, Springfield, VA 22161; (703) 487-4650. Rush orders may be placed for an extra fee by calling (800) 336-4700. The report is also available on microfiche at no charge. Refer to MFS27070 .

\section{Designing echelle spectrographs}

The echelle spectrograph design aid program (EGRAM) aids in the design of spectrographic systems that utilize echelle/first-order cross-disperser combinations. This optical combination causes a 2$D$ echellogram to fall on a detector. EGRAM describes the echellogram with enough detail to enable the user to judge effectively the feasibility of the spectrograph design. By iteratively altering system parameters, the desired echellogram can be achieved without making a physical model. EGRAM calculates system parameters that are accurate to the first order and compare favorably to results continued on page 4730 